\title{
Negative spin-exchange splitting in the exciton fine structure of AIN
}

Martin Feneberg, María Fátima Romero, Benjamin Neuschl, Klaus Thonke, Marcus Röppischer, Christoph Cobet, Norbert Esser, Matthias Bickermann, and Rüdiger Goldhahn

Citation: Appl. Phys. Lett. 102, 052112 (2013); doi: 10.1063/1.4790645

View online: https://doi.org/10.1063/1.4790645

View Table of Contents: http://aip.scitation.org/toc/apl/102/5

Published by the American Institute of Physics

\section{Articles you may be interested in}

Origins of optical absorption and emission lines in AIN

Applied Physics Letters 105, 111104 (2014); 10.1063/1.4895786

Homoepitaxial AIN thin films deposited on m-plane ( 1100) AIN substrates by metalorganic chemical vapor deposition

Journal of Applied Physics 116, 133517 (2014); 10.1063/1.4897233

Optical properties of magnesium doped $\mathrm{Al}_{\mathrm{X}} \mathrm{Ga}_{1-\mathrm{x}} \mathrm{N}(0.61 \leq \mathrm{X} \leq 0.73)$

Journal of Applied Physics 116, 143103 (2014); 10.1063/1.4897449

Vacancy compensation and related donor-acceptor pair recombination in bulk AIN

Applied Physics Letters 103, 161901 (2013); 10.1063/1.4824731

The growth and optical properties of large, high-quality AlN single crystals

Journal of Applied Physics 96, 5870 (2004); 10.1063/1.1801159

Inversion of absorption anisotropy and bowing of crystal field splitting in wurtzite $\mathrm{MgZnO}$

Applied Physics Letters 108, 221105 (2016); 10.1063/1.4953159

\section{HIDEN}

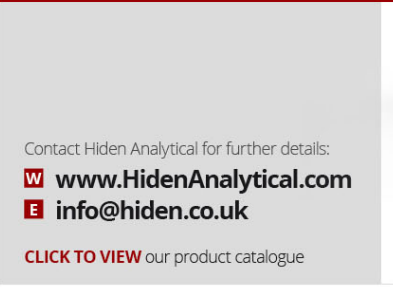

\section{Instruments for Advanced Science}

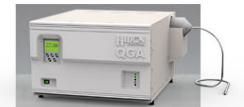

Gas Analysis

- dynamic measurement of reaction gas streams cataysis and thermal analysis

molecuar beam studes

dissolved species probes

fermentation, envirormental and ecological studis

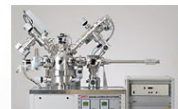

Surface Science

UHVTPD

, SIMS

, end point detection in ion beam etch , elemental imaging-surface mapping

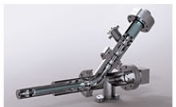

Plasma Diagnostics plasma source characterization etch and deposition process reaction kinetic studies

analysis of neutral and radical species

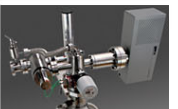

Vacuum Analysis partial pressure measurement and control of process gases , reactive sputter process control vacuum diagnostic 


\title{
Negative spin-exchange splitting in the exciton fine structure of AIN
}

\author{
Martin Feneberg, ${ }^{1, a)}$ María Fátima Romero, ${ }^{1}$ Benjamin Neuschl, ${ }^{2}$ Klaus Thonke, ${ }^{2}$ \\ Marcus Röppischer, ${ }^{3}$ Christoph Cobet, ${ }^{3}$ Norbert Esser, ${ }^{3}$ Matthias Bickermann, ${ }^{4, b)}$ \\ and Rüdiger Goldhahn ${ }^{1}$ \\ ${ }^{1}$ Institut für Experimentelle Physik, Otto-von-Guericke-Universität Magdeburg, Universitätsplatz 2, \\ 39106 Magdeburg, Germany \\ ${ }^{2}$ Institut für Quantenmaterie/Gruppe Halbleiterphysik, Universität Ulm, 89069 Ulm, Germany \\ ${ }^{3}$ Leibniz-Institut für Analytische Wissenschaften - ISAS - e.V., Albert-Einstein-Str. 9, 12489 Berlin, Germany \\ ${ }^{4}$ Department Werkstoffwissenschaften, Materialien für die Elektronik und Energietechnologie, \\ Friedrich-Alexander-Universität Erlangen-Nürnberg, Martensstr. 7, 91058 Erlangen, Germany
}

(Received 2 January 2013; accepted 24 January 2013; published online 5 February 2013)

\begin{abstract}
The exact energy position of the free exciton transition and thus the lowest band gap in bulk wurtzite AlN are still under discussion. By combined high resolution optical emission and absorption experiments on a sample with (1100) surface, we resolve the fine structure of the lowest energy free exciton and determine an electron-hole spin-exchange interaction constant of $j=-4 \mathrm{meV}$. This results in a low energy $\Gamma_{1}$ exciton at $6.032 \mathrm{eV}$ and a high energy $\Gamma_{5}$ component at $6.040 \mathrm{eV}$. Only the latter one is observable for (0001) oriented AIN films due to selection rules. (C) 2013 American Institute of Physics. [http://dx.doi.org/10.1063/1.4790645]
\end{abstract}

The fundamental excitations of semiconductors (excitons) have been investigated for decades in all kinds of materials. They consist basically of electrostatically bound electron-hole pairs and dominate optical interband transitions as long as their binding energy is larger than $\approx k_{B} T$. The exciton fine structure in hexagonal wurtzite semiconductors is linked to the valence band structure with three valence bands which are split by crystal field $\left(\Delta_{\mathrm{cf}}\right)$ and spin-orbit $\left(\Delta_{\mathrm{so}}\right)$ interaction. Additionally, the short range spin-exchange interaction leads to a further splitting of excitonic states. The corresponding exciton Hamiltonian can be written as ${ }^{1}$

$$
H^{e x c}=E_{\mathrm{X}}+\frac{1}{2} j\left(\sigma_{h} \cdot \sigma_{e}\right)
$$

Here, $E_{\mathrm{X}}$ is the exciton binding energy taking into account the corresponding valence band dispersion, $j$ is the spinexchange interaction constant, and $\sigma_{e, h}$ are spin-Pauli matrices operating on valence-electron and hole spin functions, respectively. In a simplified picture, the spin-exchange interaction $j$ amounts always to a certain fraction of $\approx 10 \%$ of the exciton binding energy ${ }^{2} E_{\mathrm{X}}$. A comprehensive table for a variety of semiconductors is provided, e.g., in Ref. 3. To study the exciton states split by spin-exchange interaction experimentally, it is natural to chose a material having a large $j$ resulting in a large separation of the different exciton states. Such materials therefore also should fulfill the criteria of a large $E_{\mathrm{X}}$.

$\mathrm{ZnO}$ has a large $E_{\mathrm{X}}$ of around $60 \mathrm{meV}$ and a large $j=5.6 \mathrm{meV}^{4}$ Alas, in this case, the valence band spacing from the highest valence band to the next one is $4.5 \mathrm{meV}$ only, much lower than the undisturbed distance between the excitons split by spin-exchange interaction of $2 j=11.2 \mathrm{meV}$.

\footnotetext{
${ }^{a}$ Author to whom correspondence should be addressed. Electronic mail: martin.feneberg@ovgu.de.

${ }^{b}$ Present address: Leibniz Institute for Crystal Growth, Max-Born-Strasse 2, 12489 Berlin, Germany.
}

This means that the intermixing between the excitons formed from holes of the different valence bands makes any investigation complicated in $\mathrm{ZnO}$. Also, $\mathrm{GaN}$ has a relatively large $E_{\mathrm{X}}$ of about $25 \mathrm{meV}$; however, its spin-exchange is reported to be as low as $j \approx 1 \mathrm{meV},{ }^{3,5,6}$ clearly violating the $\approx 10 \%$ rule-of-thumb.

Another candidate material having large $E_{\mathrm{X}}$ and additionally large separation of the highest valence bands is wurtzite AlN which has recently gained much attention due to its interesting valence band structure ${ }^{7}$ and its technological importance as upper end of the III-nitride alloy system which is, e.g., used for ultraviolet light emitters. ${ }^{8-10}$ In AlN, the exciton binding energy is consistently reported to be around $53 \mathrm{meV}$; however, spin-exchange interaction has so far not been considered to interpret spectra, which leads to the situation that the identities of observed free exciton transitions are ambiguous.

Here, we shed light on the free exciton fine structure of wurtzite AlN by a combined high resolution emission and absorption study on a sample which has the optical axis inplane. By taking fundamental symmetry considerations into account, we unambiguously identify the lowest exciton to have $\Gamma_{1}$ symmetry and an additional exciton $8 \mathrm{meV}$ higher in energy with $\Gamma_{5}$ symmetry. This order of the exciton states yields a negative value for the spin-exchange interaction of $j=-4 \mathrm{meV}$, what was not observed for any bulk semiconductor material before.

Due to its large negative crystal field splitting of $\Delta_{\mathrm{cf}}=-221 \mathrm{meV}$, the valence band order of AlN is $\Gamma_{7}, \Gamma_{9}, \Gamma_{7}$. The value of $\Delta_{\mathrm{cf}}$ is approximately the splitting between the highest and the other two valence bands, which in turn are just split by the much lower spin-orbit interaction $\left(\Delta_{\mathrm{so}}=13 \mathrm{meV}\right)$. These conditions allow for a detailed analysis of the optical properties of the wurtzite material system, including their anisotropic symmetry characteristics.

On high quality (0001) $c$-oriented homoepitaxial layers, an energy of $6.040 \mathrm{eV}$ is reported at low temperature for the lowest free exciton transition, ${ }^{11-13}$ while on off-oriented 
layers a slightly lower value is found ${ }^{14,15}$ (see also the discussion in Ref. 11). From samples with non- and semipolar surfaces, however, energies at or below $6.035 \mathrm{eV}$ are found. ${ }^{16-18}$ These differences cannot be explained by changes in strain and/or impurity concentrations alone. In the following, we will show that a natural and physical interpretation is possible in terms of spin-exchange splitting by distinguishing between $\Gamma_{1}$ and $\Gamma_{5}$ excitons.

Electrons from the $\Gamma_{7}$ conduction band form excitons when interacting with holes from the $\Gamma_{7}$ valence band. Thus, excitons of $\Gamma_{1}, \Gamma_{2}$, and $\Gamma_{5}$ symmetry in $\mathrm{C}_{6 \mathrm{v}}$ are formed, where the latter are twofold degenerate

$$
\Gamma_{7} \otimes \Gamma_{7} \rightarrow \Gamma_{1}+\Gamma_{2}+\Gamma_{5}(2) .
$$

$\Gamma_{2}$ states are dipole forbidden paraexcitons and cannot contribute to optical spectra, thus we are left with the $\Gamma_{1}$ and $\Gamma_{5}$ symmetry states forming orthoexcitons. In our case, the polarization orientation of the electric field vector for each exciton state is guiding their unambiguous identification. The $\Gamma_{1}$ exciton is expected to be only visible for $\mathbf{E} \| \mathbf{c}$ and $\mathbf{k} \perp \mathbf{c}$, the $\Gamma_{5}$ exciton only for $\mathbf{E} \perp \mathbf{c}$ and $\mathbf{k} \| \mathbf{c}$ or $\mathbf{k} \perp \mathbf{c}$ but with much lower oscillator strength.

The degeneracy of $\Gamma_{1}$ and $\Gamma_{5}$ states is lifted by the nonvanishing electron-hole spin-exchange interaction $j$. We calculated the resulting exciton fine structure by diagonalizing

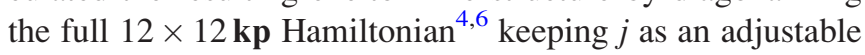
parameter. As long as we are concerned with experimental situations with $\mathbf{E}, \mathbf{k}$ along principal axes, no further splitting in transverse and longitudinal branches due to the polaritonic nature of the excitons is expected to be observable.

The sample under study is a high quality AlN single crystal grown by physical vapor transport. ${ }^{19}$ Its surface is (1100) oriented within an accuracy of $\pm 0.3^{\circ}$. The sample is about $500 \mu \mathrm{m}$ thick. Lattice constants are $c=4.9810 \AA$ and $a=3.1109 \AA$ according to XRD measurements. Chemical analysis of a companion sample yielded impurity concentrations of $[\mathrm{O}]=7 \times 10^{18} \mathrm{~cm}^{-3},[\mathrm{Si}]=5 \times 10^{18} \mathrm{~cm}^{-3}$, and $[C]=6 \times 10^{17} \mathrm{~cm}^{-3}$. Photoluminescence was excited by laser pulses of an $\mathrm{ArF}^{*}$ excimer laser with a wavelength of $\lambda=193 \mathrm{~nm}$ in the low excitation regime. An $\alpha$-BBO (barium borate) Rochon polarizer was used to selectively record different polarization directions of the electric field vector. Luminescence light was dispersed by a grating monochromator with $1.25 \mathrm{~m}$ focal length and detected by a liquid nitrogen cooled CCD camera. The spectral resolution of our setup was better than $250 \mu \mathrm{eV}$ at $6 \mathrm{eV}$. For reflectivity spectra, a $\mathrm{D}_{2}$ lamp was used as light source, while as reference, a special high UV reflectivity mirror was employed. By spectroscopic ellipsometry, the parameters $\Psi$ and $\Delta$ were recorded for caxis orientation parallel and perpendicular to the plane of incidence. ${ }^{20}$ The data analysis yielded the ordinary $(\mathbf{E} \perp \mathbf{c})$ and extra-ordinary $(\mathbf{E} \| \mathbf{c})$ dielectric functions $\left(\bar{\varepsilon}_{j}=\varepsilon_{1, j}+i \varepsilon_{2, j}\right.$; $j=\perp, \|)$ which were then analyzed ${ }^{21}$ with respect to the excitonic contributions. The high resolution of around $1 \mathrm{meV}$ at $6 \mathrm{eV}$ necessary for this study was achieved by accessing the ellipsometer attached to the Berlin electron storage ring for synchrotron radiation (BESSY II).

The imaginary parts of the anisotropic dielectric function at low temperature are presented in Fig. 1. These spectra

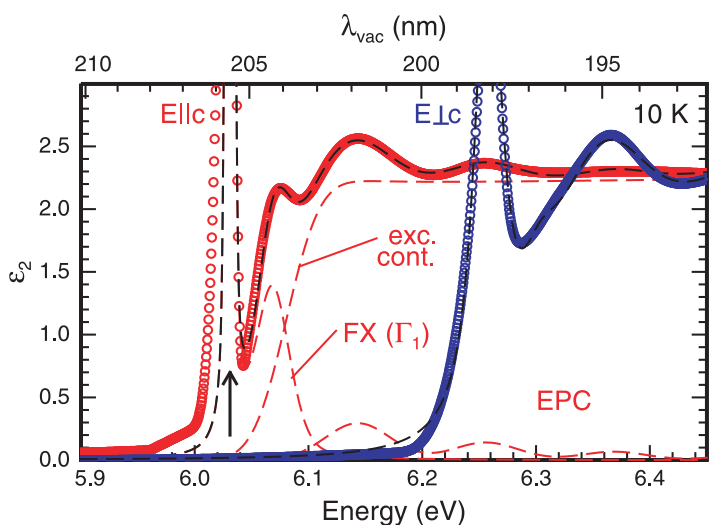

FIG. 1. Imaginary parts of the dielectric function around the band gap at $T=10 \mathrm{~K}$. The ordinary (blue) and extra-ordinary (red) dielectric functions are shown (open circles) together with a model line shape fit (dashed black line). For the extraordinary dielectric function, the single components adding up to $\varepsilon_{2}$ are shown (red dashed curves). The black arrow marks the resonance energy of $6.032 \mathrm{eV}$. Please note that the peak maximum of $\varepsilon_{2}$ at $\mathbf{E} \| \mathbf{c}$ is at 17 . The fit (dashed line) is chosen such to match the peak maximum.

are truncated to clearly visualize the details around the fundamental absorption edge. For $\mathrm{E} \| \mathrm{c}\left(\varepsilon_{2, \|}\right)$, the multiple components (as described earlier ${ }^{22}$ ) contributing to the absorption are indicated. We find a very satisfying agreement of fitted and experimental spectrum for an exciton resonance peaking at $6.032 \mathrm{eV}$ (amplitude is about 17) and additional excited states within a simple hydrogen model with an exciton binding energy of $53 \mathrm{meV}$ (labeled $\mathrm{FX}\left(\Gamma_{1}\right)$ ). The exciton continuum (exc. cont.) and exciton-phonon complexes (EPCs) are also visible clearly. Both are compatible with an exciton binding energy of $53 \mathrm{meV}$ and a longitudinal optical phonon energy of $110 \mathrm{meV}$, respectively. For $\mathbf{E} \perp \mathbf{c}$, the imaginary part of the dielectric function is shifted to a higher energy onset. Exciton resonances belonging to the $\Gamma_{5}$ symmetry are found at $6.255 \mathrm{eV}$ and $6.264 \mathrm{eV}$ yielding $\Delta_{\mathrm{cf}}=-221 \mathrm{meV}$ and $\Delta_{\mathrm{so}}=13 \mathrm{meV}$.

From the complete dielectric function, we calculated a normal incidence reflectivity spectrum and compared it to an experimentally obtained one (Fig. 2). Both curves show identical features at the same energy values confirming the validity of our dielectric function. Minor differences occur

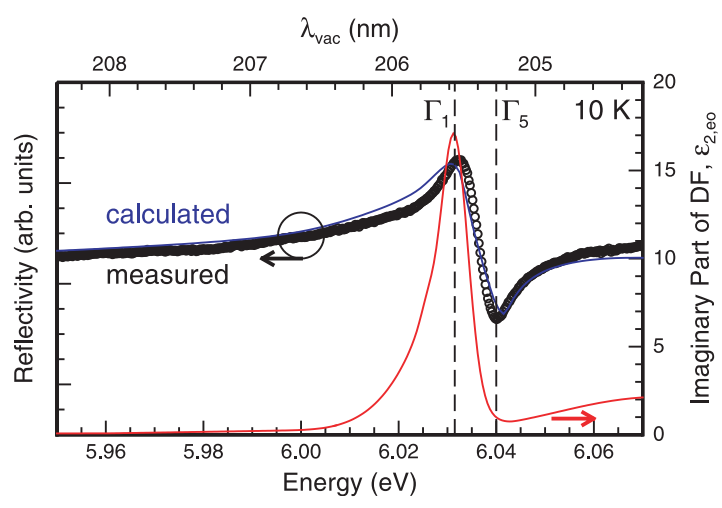

FIG. 2. Reflectivity calculated from the measured dielectric function (blue, continuous curve) in comparison to experimentally determined reflectivity (black, open circles). Remaining differences are mainly due to the better spectral resolution of the experiment implying the $\mathrm{D}_{2}$ lamp. The imaginary part of the dielectric function $\varepsilon_{2, e o}$ is shown for comparison (red, this is the same spectrum as already shown in Fig. 1). 
due to the higher spectral resolution of the spectra obtained with the $\mathrm{D}_{2}$ lamp. A simple Lorentz oscillator fit of the reflectivity curves yields a too high transition energy compared to the peak from $\varepsilon_{2}$ located at $6.032 \mathrm{eV}$. This is due to a negative $\varepsilon_{1}$ close to the exciton resonance (not shown here) and the very high value of $\varepsilon_{2} \approx 17$ at the resonance energy.

Photoluminescence spectra were taken in different geometries from our sample. Fig. 3 shows a comparison of (normalized) spectra taken from different surfaces and in different polarization orientations. First, we recorded a spectrum from the (0001) side-surface of our $500 \mu \mathrm{m}$ thick sample (k\| $\mathbf{c}, \mathbf{E} \perp \mathbf{c}$, blue trace). We find a single free exciton contribution peaking at $6.040 \mathrm{eV}$. The same peak is visible also in a spectrum taken from the $(1 \overline{1} 00)$ surface plane with the electric field vector oriented parallel to (0001) ( $\mathbf{k} \perp \mathbf{c}, \mathbf{E} \| \mathbf{c}$, red trace). However, an additional contribution at $6.032 \mathrm{eV}$ becomes visible as well, exactly at the same energy position where the imaginary part of the extraordinary dielectric function has its maximum (also shown in Fig. 3 as trace labelled $\varepsilon_{2}$ ). This exciton visible in $\mathbf{E} \| \mathbf{c}$ is unambiguously identified as $\Gamma_{1}$ state. The higher energy component at $6.040 \mathrm{eV}$ is only visible in emission when an $\mathbf{E} \perp \mathbf{c}$ component is observable (in our $\mathbf{k} \perp \mathbf{c}, \mathbf{E} \| \mathbf{c}$ spectrum, this seems to be the case unintentionally). This contribution is thus attributed to the $\Gamma_{5}$ symmetry state.

For $\mathbf{k} \perp \mathbf{c}, \mathbf{E} \perp \mathbf{c}$ orientation, one would expect the identical spectrum as found from the ( $c$ plane) side facet of our sample. However, here we detect a relatively sharp bound exciton with maximum at $6.027 \mathrm{eV}$ obscuring the clear observation of the expected free exciton. This transition is identified as a bound exciton (presumably to a neutral donor) from temperature dependent luminescence experiments. Note that this donor bound exciton is not visible for $\mathbf{E} \| \mathbf{c}$ as already found by Funato et al. ${ }^{13}$

We can follow the transition energies of $\Gamma_{1}$ and $\Gamma_{5}$ exciton states in temperature dependent spectra and find a perfect agreement for the $\Gamma_{1}$ exciton energy obtained from luminescence and ellipsometry data (Fig. 4) further corroborating our identification. The temperature dependence of the $\Gamma_{1}$ exciton as obtained from ellipsometry is fitted by Pässler's model ${ }^{23}$ and yields the parameters $E(0)=6.032 \mathrm{eV}, \alpha=0.77 \mathrm{meV} / \mathrm{K}$,

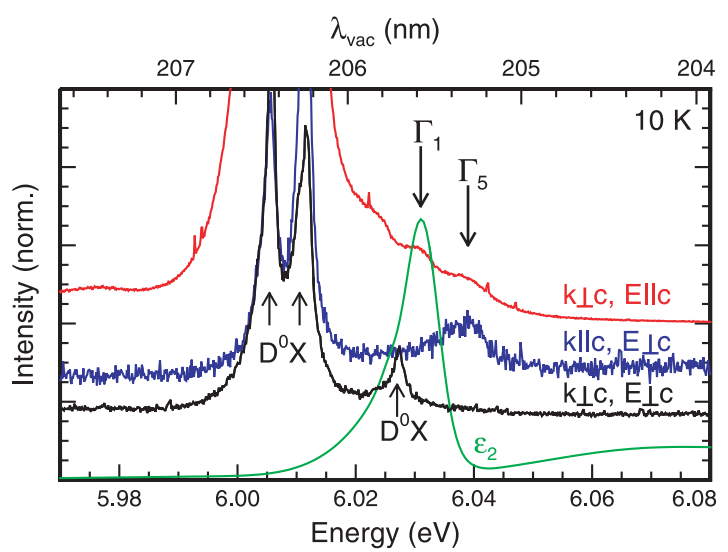

FIG. 3. Comparison of $\varepsilon_{2}$ and photoluminescence spectra under different polarization directions of the electric field vector. The scaling of the spectra is adjusted independently to make the free exciton region clearly visible. Bound exciton contributions are labelled by $\mathrm{D}^{0} \mathrm{X}$. Arrows mark the two free exciton bands at 6.032 and $6.040 \mathrm{eV}$.

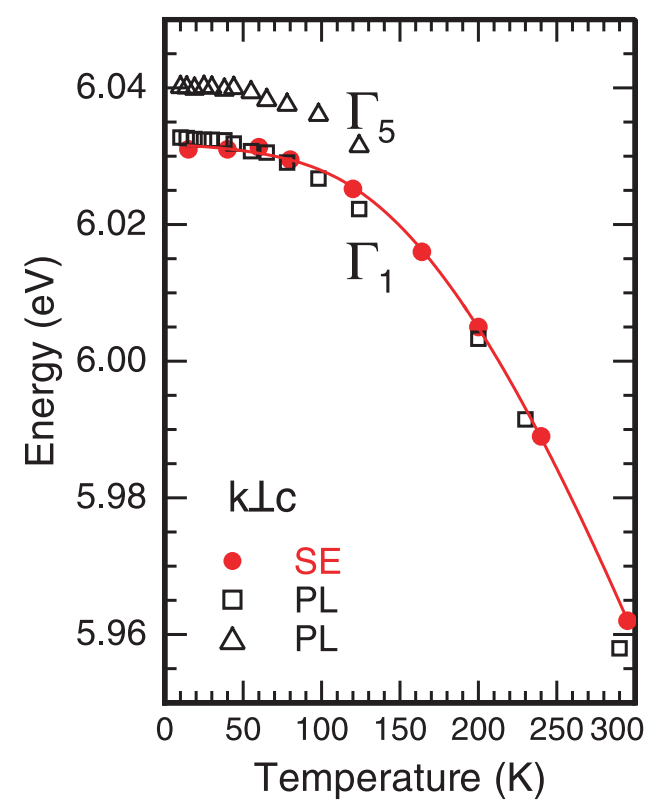

FIG. 4. Transition energies of the different free excitons measured for $\mathbf{E} \| \mathbf{c}$ as a function of temperature. Open symbols represent peak energies from PL spectra, and filled red circles are results from fitting the dielectric function. The continuous red line is the best fit to the ellipsometric data using Pässler's equation. ${ }^{23}$

$\Theta=642 \mathrm{~K}$, and $\Delta=0.30$, in good agreement with earlier studies. $^{12}$

We find that the observed splitting can be best represented by an exciton ground state energy (for $j=0$ ) of $6.038 \mathrm{eV}$ and a spin-exchange interaction constant of $j=-4 \mathrm{meV}$. The splitting between $\Gamma_{1}$ and $\Gamma_{5}$ states is $2 j$, while the energy distance of the two exciton states is $\Delta E\left(\Gamma_{1}\right)=+3 j / 2$ and $\Delta E\left(\Gamma_{5}\right)=-j / 2$ in the present case of negligible mixing with further valence bands. The value for $j$ is comparably large and negative, which has not been observed for other semiconductors yet. The special intrinsic situation in wurtzite AIN opens the possibility to study a ground state $\Gamma_{1}$ free exciton with relatively large separation $(8 \mathrm{meV})$ from the $\Gamma_{5}$ counterpart and vanishing intermixing with states from other valence bands.

Finally, we want to comment on the exciton binding energy in AIN. Our ellipsometry results are well reproduced by a fit using the hydrogen model, in agreement with Gil et $a l .{ }^{24}$ who found only small anisotropic corrections necessary. The experimental value presented here is strictly speaking only valid for the $\Gamma_{1}$ component of the exciton; however, the exciton binding energy is a function of the polariton effective mass and static dielectric constants. All these parameters are not expected to differ for $\Gamma_{1}$ and $\Gamma_{5}$ excitons, which suggest that the exciton binding energy is the same for excitons of both symmetries. The lowest band gap of wurtzite $\mathrm{AlN}$ is therefore at $6.091 \mathrm{eV}, 53 \mathrm{meV}$ above the exciton ground state energy (for $j=0)$ of $6.038 \mathrm{eV}(T=10 \mathrm{~K})$.

In conclusion, we resolved a long lasting discussion about the free exciton transition in wurtzite AlN by combined high resolution spectroscopic ellipsometry and emission experiments. We clarified the corresponding fine structure of excitons resulting in a spin-exchange interaction energy of $j=-4 \mathrm{meV}$. Based on our findings, all previous optical spectroscopy results of wurtzite AIN can be put in 
perspective. The complicated properties of this material system are untangled and the way for a better comparability and collaboration between researchers in the field of AlN is paved.

We gratefully acknowledge support by the synchrotron radiation source BESSY II of the Helmholtz-Zentrum Berlin (HZB). A part of this work was supported by the EU within the 7th RTD Framework (project RAINBOW: Contract No. PITN-GA-2008-213238). Further experiments were performed at Beamline I of Hasylab, DESY (Hamburg, Germany). The access to the beamline and support is gratefully acknowledged.

${ }^{1}$ O. Akimoto and H. Hasegawa, Phys. Rev. Lett. 20, 916 (1968).

${ }^{2}$ P. G. Rohner, Phys. Rev. B 3, 433 (1971).

${ }^{3}$ M. Julier, J. Campo, B. Gil, J. P. Lascaray, and S. Nakamura, Phys. Rev. B 57, R6791 (1998).

${ }^{4}$ D. W. Langer, R. N. Euwema, K. Era, and T. Koda, Phys. Rev. B 2, 4005 (1970).

${ }^{5}$ P. P. Paskov, T. Paskova, P. O. Holtz, and B. Monemar, Phys. Rev. B 64, 115201 (2001).

${ }^{6}$ R. Ishii, A. Kaneta, M. Funato, Y. Kawakami, and A. A. Yamaguchi, Phys. Rev. B 81, 155202 (2010).

${ }^{7}$ L. Chen, B. J. Skromme, R. F. Dalmau, R. Schlesser, Z. Sitar, C. Chen, W. Sun, J. Yang, M. A. Khan, M. L. Nakarmi, J. Y. Lin, and H.-X. Jiang, Appl. Phys. Lett. 85, 4334 (2004).

${ }^{8}$ Y. Taniyasu, M. Kasu, and T. Makimoto, Nature (London) 441, 325 (2006).
${ }^{9}$ T. Oto, R. G. Banal, K. Kataoka, M. Funato, and Y. Kawakami, Nat. Photonics 4, 767 (2010).

${ }^{10}$ M. Shatalov, W. Sun, A. Lunev, X. Hu, A. Dobrinsky, Y. Bilenko, J. Yang, M. Shur, R. Gaska, C. Moe, G. Garrett, and M. Wraback, Appl. Phys. Express 5, 082101 (2012).

${ }^{11}$ M. Feneberg, B. Neuschl, K. Thonke, R. Collazo, A. Rice, Z. Sitar, R. Dalmau, J. Xie, S. Mita, and R. Goldhahn, Phys. Status Solidi A 208, 1520 (2011).

${ }^{12}$ B. Neuschl, K. Thonke, M. Feneberg, S. Mita, A. Xie, R. Dalmau, R. Collazo, and Z. Sitar, Phys. Status Solidi B 249, 511 (2012).

${ }^{13}$ M. Funato, K. Matsuda, R. G. Banal, R. Ishii, and Y. Kawakami, Appl. Phys. Express 5, 082001 (2012).

${ }^{14}$ E. Silveira, J. A. Freitas, M. Kneissl, D. W. Treat, N. M. Johnson, G. A. Slack, and L. J. Schowalter, Appl. Phys. Lett. 84, 3501 (2004)

${ }^{15}$ E. Silveira, J. A. Freitas, O. J. Glembocki, G. A. Slack, and L. J. Schowalter, Phys. Rev. B 71, 041201(R) (2005).

${ }^{16} \mathrm{H}$. Murotani, T. Kuronaka, Y. Yamada, T. Taguchi, N. Okada, and H. Amano, J. Appl. Phys. 105, 083533 (2009).

${ }^{17}$ A. Sedhain, N. Nepal, M. L. Nakarmi, T. M. Al Tahtamouni, J. Y. Lin, H. X. Jiang, Z. Gu, and J. H. Edgar, Appl. Phys. Lett. 93, 041905 (2008).

${ }^{18}$ M. Feneberg, R. A. R. Leute, B. Neuschl, K. Thonke, and M. Bickermann, Phys. Rev. B 82, 075208 (2010).

${ }^{19}$ M. Bickermann, B. M. Epelbaum, O. Filip, P. Heimann, S. Nagata, and A. Winnacker, Phys. Status Solidi C 7, 21 (2010).

${ }^{20}$ C. Cobet, R. Goldhahn, W. Richter, and N. Esser, Phys. Status Solidi B 246, 1440 (2009).

${ }^{21}$ R. Goldhahn, Acta Phys. Pol. A 104, 123 (2003).

${ }^{22}$ G. Rossbach, M. Feneberg, M. Röppischer, C. Werner, N. Esser, C. Cobet, T. Meisch, K. Thonke, A. Dadgar, J. Bläsing, A. Krost, and R. Goldhahn, Phys. Rev. B 83, 195202 (2011).

${ }^{23}$ R. Pässler, Phys. Rev. B 66, 085201 (2002).

${ }^{24}$ B. Gil, D. Felbacq, B. Guizalm, and G. Bouchitté, Phys. Status Solidi B 249, 455 (2012). 